\title{
Frontières
}

\section{Célébrer la mort ?}

\section{Mélancolie et représentation}

\section{Daniel Castillo Durante}

Volume 18, numéro 1, automne 2005

Hélas, célébrer la mort!

URI : https://id.erudit.org/iderudit/1074307ar

DOI : https://doi.org/10.7202/1074307ar

Aller au sommaire du numéro

Éditeur(s)

Université du Québec à Montréal

ISSN

1180-3479 (imprimé)

1916-0976 (numérique)

Découvrir la revue

Citer ce document

Castillo Durante, D. (2005). Célébrer la mort ? Mélancolie et représentation.

Frontières, 18(1), 6-7. https://doi.org/10.7202/1074307ar

Ce document est protégé par la loi sur le droit d'auteur. L'utilisation des services d'Érudit (y compris la reproduction) est assujettie à sa politique d'utilisation que vous pouvez consulter en ligne.

https://apropos.erudit.org/fr/usagers/politique-dutilisation/
Cet article est diffusé et préservé par Érudit.

Érudit est un consortium interuniversitaire sans but lucratif composé de l’Université de Montréal, l'Université Laval et l'Université du Québec à Montréal. Il a pour mission la promotion et la valorisation de la recherche. https://www.erudit.org/fr/ 


\section{CÉLÉBRER LA MORT ? MÉLANCOLIE ET REPRÉSENTATION}

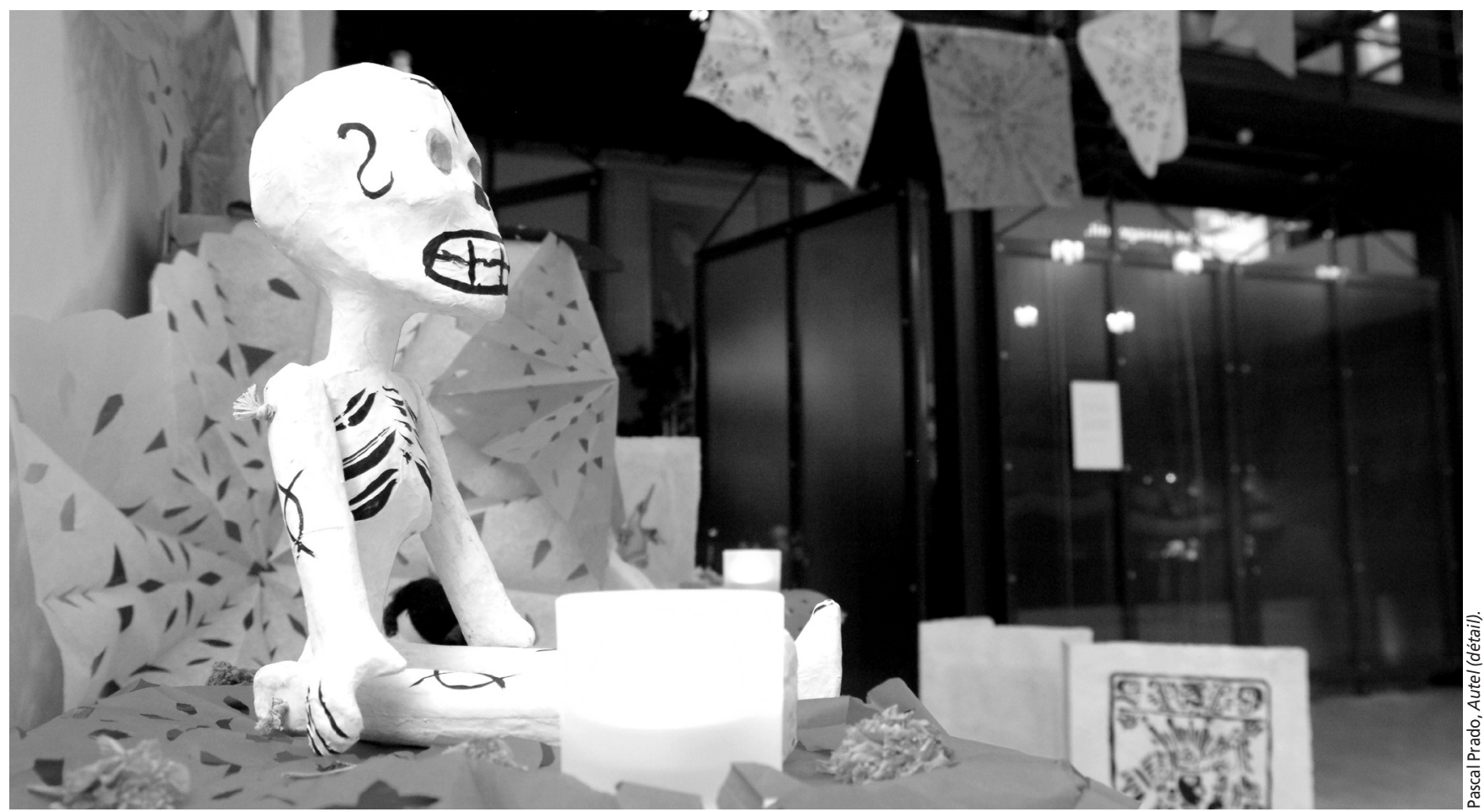

Daniel Castillo Durante, Ph. D., responsable du thème.

Depuis la nuit des temps, l'idée de mourir - beaucoup plus que le phénomène en tant que tel - nous effraie. On dirait que l'image mentale dans laquelle nous enfermons notre notion de la mort constitue le premier obstacle à une compréhension juste, sereine et lucide du phénomène. Mais peut-on comprendre la mort? Acceptons-nous vraiment au plus profond de notre conscience le mécanisme inexorable qui la sous-tend? Est-il possible ne serait-ce que d'imaginer notre conscience désertée à tout jamais par le souffle qui la maintenait jusque-là rivée à la vie? Lorsque j'étais enfant, la nuit dans mon lit, il m'arrivait parfois de «jouer» à être mort et je me rappellerai toujours le frisson d'épouvante et de sublime à la fois qui se déclenchait en moi à ces momentslà. Tout se passait comme si l'épreuve simulée de la mort célébrait en même temps le fait d'être né à la vie. Plus tard, j'ai essayé de revisiter l'imaginaire qui était alors le mien et je me suis aperçu que notre société de consommation effrénée et d'exploitation médiatique d'une représentation frelatée et commerciale de la mort m'en empêchait l'accès. J'en suis venu à la conclusion que notre construction occidentale à gros coups de stéréotypes de ce lieu commun qu'est la mort la subordonnait aux lois du marché en en faisant une marchandise paradigmatique. La «mort» se porte bien et elle rapporte beaucoup d'argent. Voilà un constat cinglant à l'heure des mondialisations des économies. Devrait-on aussi parler de mondialisation de la mort? Les différents discours médiatiques ainsi que les pratiques culturelles (cinéma, littérature, théâtre, arts visuels, etc.) privilégient une représentation de la mort associée à la violence, au crime, à la guerre, au terrorisme, aux catastrophes naturelles, aux maladies et aux distinctes formes de prédation sexuelle qui aboutissent à l'extinction de la vie des victimes.

La mort comme cycle en interaction, voire en dialogue avec la naissance ${ }^{1}$ échappe à notre imaginaire occidental qui la voit immanquablement en tant que rupture, intrusion, hiatus, divorce, incompatibilité foncière, altérité radicale. Cela explique sans doute le fait que, pour les Occidentaux, l'opposé de la mort ne soit que la vie. Une société ne valorisant que le matérialisme, la nôtre, développe ainsi un attachement à la «vie» en fonction d'un lien de pure consommation qui nous prive du registre tellurique, si j'ose dire, à partir duquel la «fin de la naissance» prend tout naturellement sa place dans l'ordre des choses. Dans ce contexte, cesser de naître, nous dé-prendre en quelque sorte de notre venue au monde, serait le signe final de notre passage sur terre. La vie que nous habitons s'y révèle donc un prêt dont nous sommes responsables au nom de la communauté des mortels beaucoup plus qu'à titre individuel. L'individualisation de la mort si caractéristique de nos sociétés capitalistes postmodernes explique en grande partie le déclin des rituels à l'heure actuelle. La 
mort individuelle y est éprouvée exclusivement comme une perte. S'efface ainsi le caractère collectif de l'événement. La mort en tant qu'évolution ultime de la naissance peut donner lieu à une re-naissance si l'on est croyant ou à une expiration pure et simple des délais du prêt vital qui devrait être perçu comme un don émanant forcément d'un souffle collectif. La crispation sur l'avoir au détriment de l'être cède ici la place à une dé-prise qui est également le dernier avatar de toute naissance. La mort, son apostrophe muette, apparaît dès lors comme une dernière naissance. Dans le Mexique pré-hispanique, la mort est liée à la fertilité de la terre. D'où les différents rituels agricoles qui, le jour des Morts (el día de muertos $^{2}$ ), inscrit la fin de l'existence humaine dans un cycle de naissance et de re-naissance moyennant lequel la terre nourricière se renouvelle ${ }^{3}$. La culture mexicaine de la mort voit dans la collectivité la passerelle nécessaire entre deux phénomènes - naissance et crépuscule - dont la terre fait des saisons qui encadrent les pratiques humaines. C'est à la lumière de cette réflexion que le présent numéro a pris son essor, en réunissant des chercheurs et des artistes autour de la notion de "célébration». Il s'agissait tout d'abord de prendre la mesure des limites de la représentation de la mort telle que nous la pratiquons de nos jours. D'où également la perspective comparatiste de plusieurs de nos articles qui s'efforcent de comprendre la mort depuis son rapport au sens (Raymond Lemieux) jusqu'à la métaphore d'une caresse posthume (Dominique Malaterre) qu'elle peut représenter lorsqu'elle s'incarne en photo. Qu'on le veuille ou non, c'est dans le cadre d'une représentation que la mort installe ses pénates en Occident. Voilà pourquoi les arts en général et la parole littéraire en particulier devaient être convoqués afin d'établir des liens entre la société et le sens de la mort au sein de nos sociétés inféodées au primat de la rentabilité immédiate. Les articles de Maxime Prévost, de Julie Delorme et de Lydia Lamontagne explorent ainsi les enjeux posés par la littérature dans son rapport à la mort. Jean-Michel Vidal, à l'aide d'exemples pris en Occident et en Afrique de l'Ouest (principalement chez les Dogons du Mali), interprète - à travers les «logiques» des cycles de vie - certaines caractéristiques relatives aux concepts de naissance, de jeunesse, de vieillesse et de mort. Françoise Roy, mythologue et écrivaine, se penche sur la renaissance spirituelle en évoquant Éros et Thanatos à l'aide d'un regard lucide sur le rôle joué par le symbolisme classique dans notre approche de la mort. Denis Bachand, fin analyste des médias, étudie avec perspicacité la spectacularisation de la mort sur nos écrans. Christian Milat, grâce à ses recherches sur l'alchimie, nous invite à voir la mort comme une célébration lorsqu'elle est dépouillée de toute portée funèbre en vue d'une immortalité posée dès lors comme l'objectif ultime du Grand Euvre. Éve DeGarie-Lamanque, porte un regard attentif sur les œuvres de Christian Boltanski et de Betty Goodwyn afin de nous aider à saisir les liens entre mémoire et survivance dans l'art contemporain. Claire Varin fait avancer son deuil de l'ami disparu en logeant sa mémoire dans un autel des morts que son voyage mexicain exhume d'un lointain passé. Par la voix de Pascale Rafie, c'est la poésie qui permet de dire d'autres visages de la mort. Sur un terrain plus près de l'intervention, le témoignage de Nicole Bouchard décrit l'émergence de nouvelles ritualités, celui de Patricia Saint-Cyr confirme la portée de ceux qui font partie d'une tradition qui nous est familière.

Raymond Lemieux, dans son article, affirme que la célébration de la mort, quel que soit le rituel impliqué, apparaît une «pure absurdité, puisque cela revient à célébrer par des signes l'insignifiance de tout signe: qu'est-ce qu'un signe s'il n'y a plus personne pour l'interpréter?». Il appert justement que la célébration mexicaine de la mort en mettant en lumière le sujet collectif réussit à penser ce lieu commun autrement que par la perte. Il n'en demeure pas moins que Raymond Lemieux a sans doute raison, dans le cadre de nos cultures occidentales assujetties à un processus d'individualisation crispée des consciences, de souligner que le «décès d'un être humain [...] c'est un événement social qui représente l'irruption d'un insensé dans l'ordre du sens ». En effet, notre société - bâtie sur la dialectique perte/mélancolie ${ }^{4}$ est toujours en quête de l'objet disparu. Cette tentative exige de sa part beaucoup d'énergie qui peut se traduire en violence. En ce sens, les pratiques artistiques (certains de nos articles le prouvent dans le présent numéro) relèvent de cette dynamique. La tradition aristotélicienne associe la mélancolie au désir. Les romantiques allemands (Schelling, Schlegel) y voyaient le sentiment à l'état pur de l'existence humaine. Contrairement au désir qui trouve satisfaction, la mélancolie ouvre sur une soif inextinguible. La mélancolie devient ainsi le paradigme de l'inassouvissement. La mort (que la figure de l'exil thématise à merveille) est probablement le lieu où la mélancolie met le mieux en lumière la fracture entre le sujet et la société dans laquelle il vit. Associée inéluctablement à la perte, la mort échappe dès lors à la pensée pour devenir l'objet du stéréotype qui la transforme en pur objet de consommation au niveau des médias. Ressassée à longueur de journée sous la forme de clichés qui la camouflent et la dénaturent, elle est instrumentalisée dans le cadre d'une société qui a besoin de recycler ses peurs et sa mélancolie afin de s'abîmer dans la méconnaissance de Narcisse. Individualiste, narcissique et mélancolique, la société marchande occidentale se donne un stéréotype de la mort, avec l'illusion à peine voilée de parvenir un jour à la contrôler. Or la mort, l'autre infranchissable, à cause précisément de son caractère irréductible, fonde l'humain. Une vie dépouillée de sa mort, ce à quoi la société marchande contemporaine nous convoque de plus en plus, cesserait d'être humaine. C'est justement parce qu'on réduit la mort à une copie, à une sorte de constat de la perte, qu'il nous est impossible de la penser, donc d'en faire un dernier tremplin avant de rejoindre la communauté des voix que la terre recueille dans le silence des saisons. Tant que la mort, surtout dans le cadre de nos sociétés contemporaines, sera perçue comme impasse, déclin et fin individuelles, la révélation dont elle est porteuse demeurera scellée. Notre civilisation, dont le but ultime est de tout broyer, s'abîme ainsi dans la perte. Or lorsque tout semble perdu - voilà le paradoxe - surgit la mort comme lieu de célébration.

\section{Notes}

1. Voir à ce sujet l'article «Du banal au sublime: célébrer la mort» de Raymond Lemieux dans ce numéro.

2. Lors de l'événement «La fête des Morts mexicaine, un espace de célébration», qui a eu lieu à l'espace Dallaire Memoria du 4 au 7 novembre 2004, un autel de morts (altar de muertos) mis en place par Arturo Jacal montrait ponctuellement la logique collective inhérente aux pratiques rituelles mexicaines. Une table ronde a aussi permis d'aborder le thème "Peut-on célébrer la mort?»

3. «De semillas y muertos», Gabriela Olmos in Día de muertos. Serenidad ritual, Artes de México, numéro 62, México, 2002, p. $56-61$.

4. Sigmund Freud, «Deuil et mélancolie», Métapsychologie, Gallimard, Paris, 2002. 